\title{
Cauda Equina Involvement in Post-Radiation Lower Motor Neuron Syndrome
}

\author{
Nozomu Matsuda ${ }^{1}$, Shunsuke Kobayashi ${ }^{1}$, Hideyuki Matsumoto ${ }^{2}$, Masayo Machii ${ }^{1}$, \\ Tomoko Soeda $^{1}$ and Yoshikazu Ugawa ${ }^{1}$
}

\begin{abstract}
Post-radiation lower motor neuron syndrome (PRLMNS) is a rare neurological complication of radiation therapy and its pathogenesis is unclear. We herein report a patient with PRLMNS who developed leg weakness 17 years after craniospinal radiation as a treatment for suprasellar germinoma. The electrophysiological evaluation, via a novel magnetic stimulation method, indicated a prolonged cauda equina conduction time, suggesting focal demyelination of the nerve roots in the cauda equina. The distribution of the denervated muscles detected by magnetic resonance imaging was consistent with patchy motor nerve root lesions. These results support the hypothesis that PRLMNS originates from ischemic radiculopathy in the cauda equina.
\end{abstract}

Key words: post-radiation lower motor neuron syndrome, magnetic stimulation, cauda equina, skeletal muscle MRI, suprasellar germinoma

(Intern Med 54: 1415-1419, 2015)

(DOI: 10.2169/internalmedicine.54.4182)

\section{Introduction}

Various neurological complications can occur after radiation therapy for a malignant neoplasm, affecting both the central and peripheral nervous systems $(1,2)$. These complications can be categorized according to their onset latencies: (i) acute spinal cord infarction can develop within hours to days after high doses of radiation; (ii) sensory myelopathy may occur several months after radiation and is often mild and transient; (iii) chronic progressive myelopathy with sensorimotor and autonomic symptoms is known to develop at an average of 14 months after radiation; and (iv) postradiation lower motor neuron syndrome (PRLMNS) typically presents more than 5 years after radiation therapy with progressive leg weakness without sensory or sphincter involvement (3-6). It has been debated whether PRLMNS results from radiation damage to the anterior horn cells or to the motor nerve roots of the cauda equina. Previous studies have suggested that radiation causes ischemic radiculopathy due to small vessel vasculopathy at the level of the cauda equina $(2,7,8)$. However, because PRLMNS is so rare, its pathogenesis remains unclear.
We recently developed a new magnetic stimulation technique for measuring the cauda equina conduction time (CECT) based on a specially devised powerful coil called a magnetic augmented translumbosacral stimulation (MATS) coil $(9,10)$. This technique is clinically useful for detecting abnormalities of the nerve roots at the level of the cauda equina.

We herein report a patient who developed PRLMNS 17 years after receiving craniospinal radiation as a treatment for suprasellar germinoma. We conducted a magnetic stimulation study with the aim of identifying the lesion site. We also performed skeletal muscle magnetic resonance imaging (MRI) of the lower extremities to map the distribution of the denervated muscles.

\section{Case Report}

A 20-year-old man developed polyuria in 1979 and was admitted to a hospital in Tokyo. The endocrinological testing revealed diabetes insipidus and hypopituitarism. Cranial computed tomography detected a suprasellar mass with a contrast enhancement effect. He was diagnosed with suprasellar germinoma and was treated with vincristine and

${ }^{1}$ Department of Neurology, Fukushima Medical University, Japan and ${ }^{2}$ Department of Neurology, Japanese Red Cross Medical Center, Japan Received for publication September 27, 2014; Accepted for publication December 18, 2014

Correspondence to Dr. Nozomu Matsuda, nozomu@fmu.ac.jp 

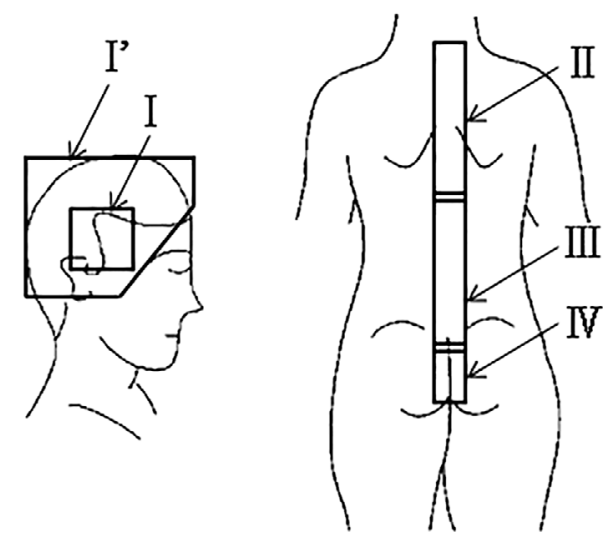

Figure 1. Irradiated areas. The patient was treated with high-dose radiation to the suprasellar region (I, 15Gy) and preventive radiation to the whole neuroaxis (I',38.6Gy; II, 38.6Gy; III, 38.6Gy) in March 1983. Additional radiation therapy was applied to the whole brain and spinal cord in January 1984 (I', 46.3Gy; II, 24.8Gy; III, 25.2Gy; IV, 27Gy).

subsequent high-dose radiation therapy to the suprasellar region (15 Gy), whole brain (84.9 Gy), and spinal cord (upper part, $63.4 \mathrm{~Gy}$; middle part, $63.8 \mathrm{~Gy}$; lower part, $27 \mathrm{~Gy}$ ) during 1983 and 1984 (Fig. 1). Complete remission was achieved and the patient continued receiving nasal desmopressin and oral levothyroxine. In 2000, he noticed intermittent claudication and had difficulty walking up stairs. The patient's leg weakness progressed gradually. On his first visit to our department in 2009, he was 50 years of age, and a physical examination revealed alopecia of the head and radiation dermatitis along the spine (Fig. 2). A neurological examination revealed that higher cognitive function was intact and the visual field was full. The cranial nerves were intact, including the bulbar function. The patient had leg weakness predominantly on the right side, but he had no weakness in the upper limbs and trunk. His muscle strength quantified on the Medical Research Council scale (right, left) was $(4,5)$ in the iliopsoas, $(4,5)$ in the quadriceps femoris, $(3,4)$ in the hamstrings, $(5,5)$ in the tibialis anterior, and $(3,4)$ in the gastrocnemius, respectively. The muscle volumes were mostly preserved except for mild atrophy in the right quadriceps femoris. The muscle tones were normal and fasciculation was not detected. Only the right ankle jerk was diminished, and bilateral plantar responses were in flexor. The patient did not complain of numbness, and all modalities of sensation were intact. He had no urinary disturbance. A laboratory examination revealed elevated serum creatine kinase levels (CK, 707 IU/L) and an elevated cerebrospinal fluid protein $(95 \mathrm{mg} / \mathrm{dL})$ without pleocytosis. Other laboratory data were normal, including thyroid function. Serum anti-ganglioside antibodies, including $\operatorname{IgM}$ antiGM1 antibody, were not detected. Nerve conduction studies revealed prolonged minimum F-wave latencies (right 56.8 $\mathrm{ms}$, left $50.6 \mathrm{~ms}$ ) and slightly reduced compound muscle action potential (CMAP) amplitudes $(7.9 \mathrm{mV}, 6.5 \mathrm{mV})$ in the tibial nerves with normal distal conduction velocities (40.9

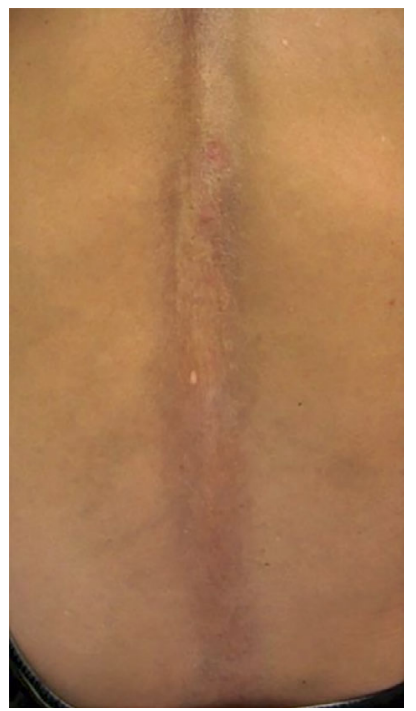

Figure 2. Radiation dermatitis. Radiation dermatitis along the spine.

$\mathrm{m} / \mathrm{s}, 41.7 \mathrm{~m} / \mathrm{s})$. Sural nerve stimulation elicited reduced sensory nerve action potentials (SNAPs) $(2.0 \mu \mathrm{V}, 1.9 \mu \mathrm{V})$, but the conduction velocities were normal $(45.2 \mathrm{~m} / \mathrm{s}, 48.6 \mathrm{~m} / \mathrm{s})$. The nerve conduction studies of the right median and ulnar nerves revealed no abnormalities. In the somatosensory evoked potential (SSEP) study with the right tibial nerve stimulation, the P38 latency was delayed to $47.6 \mathrm{~ms}$ (mean+ 2.5 SDs of the normative data corrected for body height, $43.5 \mathrm{~ms}$ ). The $\mathrm{N} 8$ onset latency was $7.2 \mathrm{~ms}$ (normal upper limit, $8.6 \mathrm{~ms}$ ). A needle electromyography (EMG) examination found motor units of high amplitude and prolonged duration with fibrillations and positive sharp waves in the right vastus lateralis, short head of the femoris biceps, tibialis anterior, and L4 paraspinal muscles. Myokymic discharges were abundant in these muscles (Fig. 3). The EMG findings were normal in the right biceps brachii and extensor digitorum communis. The lumbar MRI detected no structural abnormalities in the spinal cord or cauda equina. Contrast enhanced MRI was not performed.

We measured the CECT using a method previously described $(9,10)$. We stimulated the cauda equina at both the proximal and distal sites by placing the edge of the coil over the spinous process of the L1 and S1 vertebrae, respectively (Fig. 4). CMAPs were recorded from the right abductor hallucis. The CMAP amplitude evoked by S1 stimulation was reduced. The CECT of control populations examined in previous studies was $3.7 \pm 0.7 \mathrm{~ms}$ (mean $\pm \mathrm{SD}$ ), with an upper limit $($ mean+2.5 SDs) of $5.5 \mathrm{~ms}(9,10)$. The CECT of our patient was significantly prolonged to $6.4 \mathrm{~ms}$.

MRI of the lower extremities disclosed abnormal signals in the skeletal muscles of the buttock, thigh, and calf predominantly on the right side (Fig. 5). A conspicuous finding was that muscles even within the same myotome were often unevenly involved; for example, despite the marked T2 elongation in the right vastus lateralis and vastus intermedius, the rectus femoris and vastus medialis were mostly spared. 


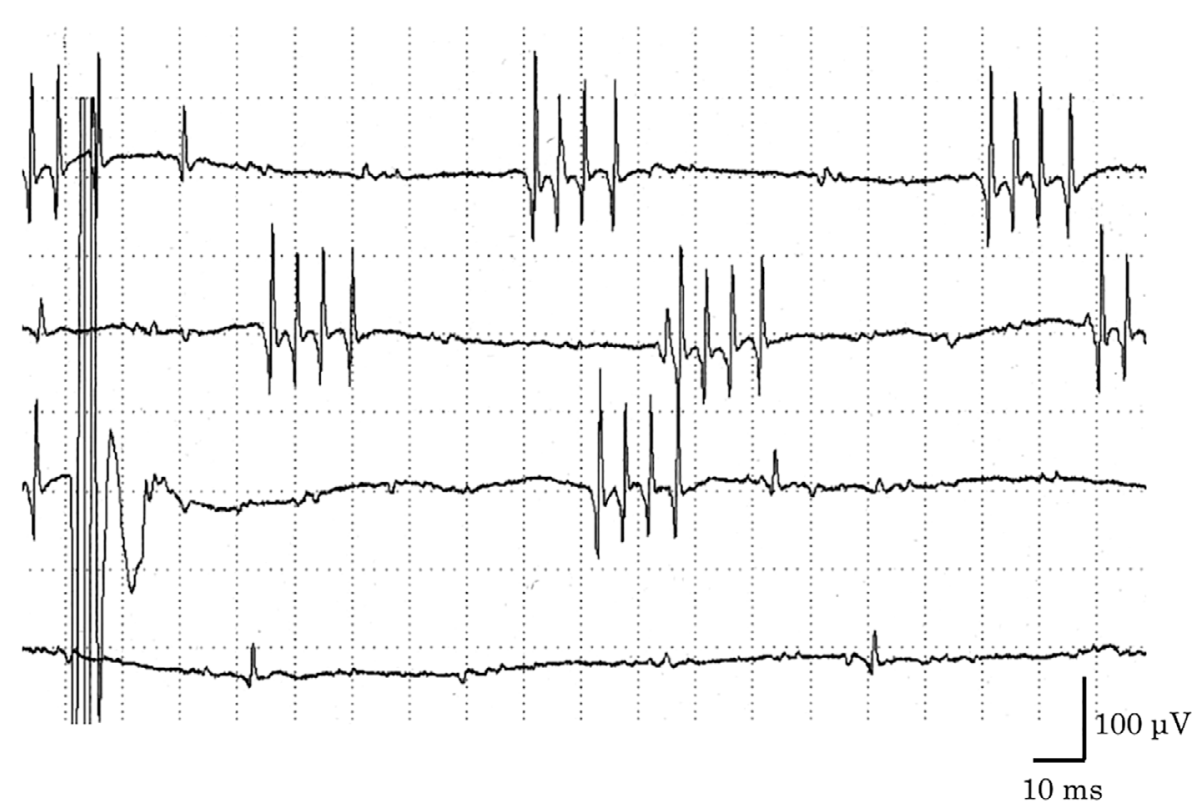

Figure 3. Myokymic EMG discharges. Myokymic discharges detected in the right tibialis anterior muscle on the needle EMG examination.

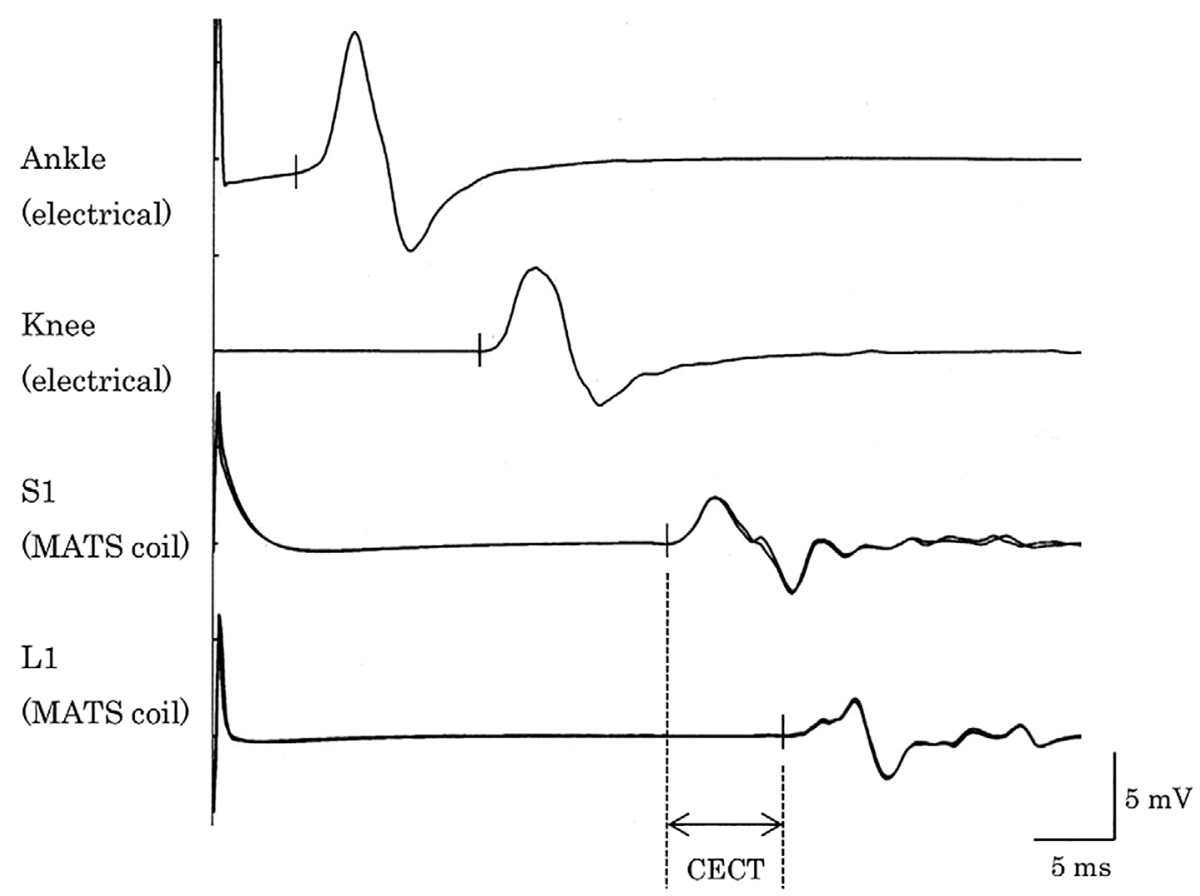

Figure 4. The electrophysiological study. The results of the motor nerve conduction studies by electric stimulation of the tibial nerve at the ankle and knee were normal (top two traces). By contrast, magnetic stimulation at the S1 and L1 levels revealed a CECT of 6.4ms (bottom two traces), which significantly exceeded the normal range $(3.7 \pm 0.7 \mathrm{~ms})$. The latencies of the compound muscle action potentials were 4.6ms (ankle), 14.6ms (knee), 24.7ms (S1), and 31.1ms (L1).

Furthermore, the long head of the right biceps femoris and semitendinosus showed T2 elongation, whereas the semimembranosus muscle appeared normal, even though all of these muscles belong to myotomes of L5, S1, and S2 and are all innervated by the sciatic nerve. The involved muscles exhibited high-intensity signals on both $\mathrm{T} 1-$ and $\mathrm{T} 2$ weighted images, but they exhibited isointense signals on short tau inversion recovery (STIR) images.

The patient's leg weakness progressed slowly, and he was required to use a walking stick in 2013. During the followup, serum CK was constantly elevated within the range of 300 to $900 \mathrm{IU} / \mathrm{L}$. 

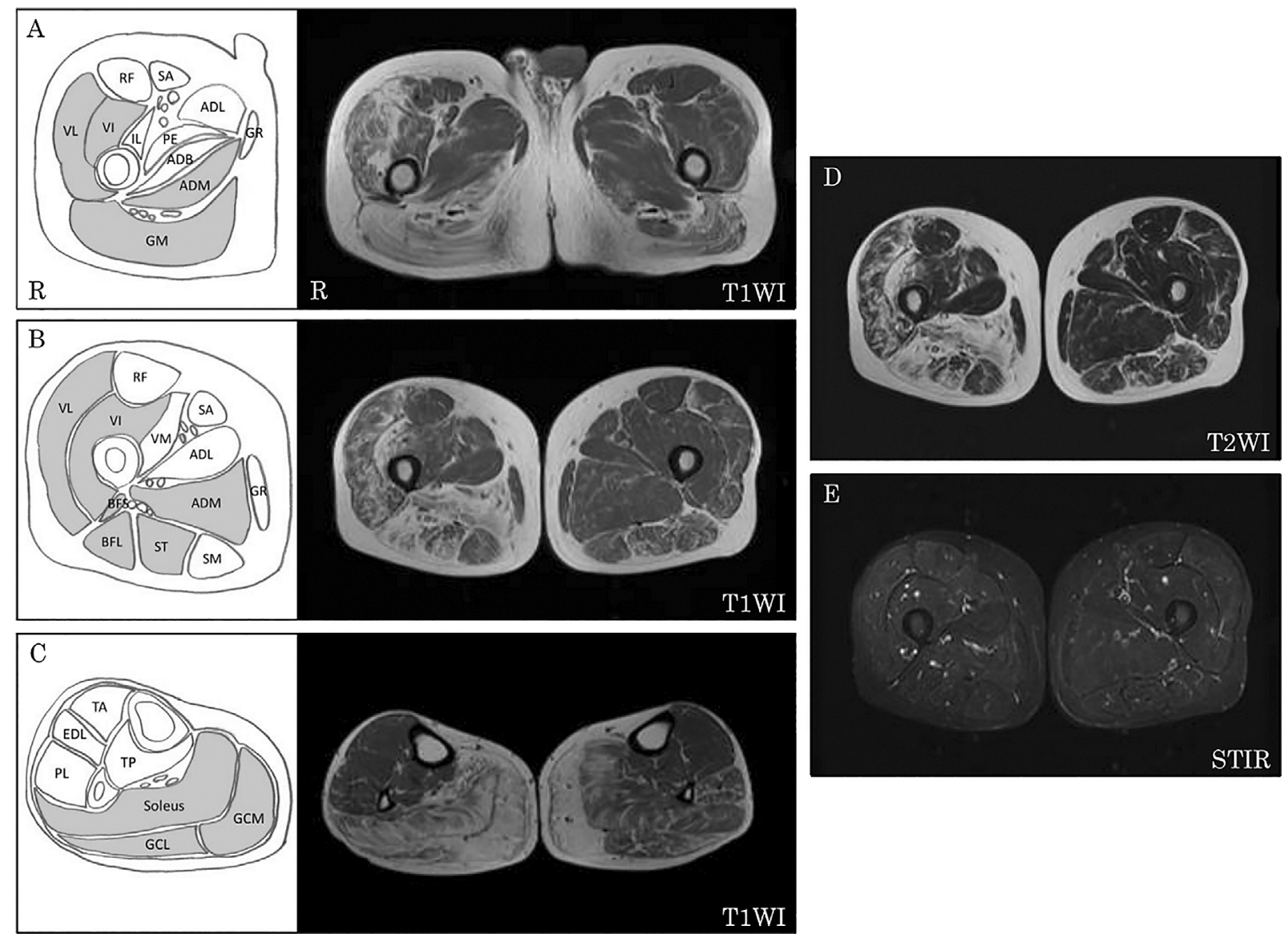

Figure 5. The skeletal muscle MRI study. T1-weighted images (TR=420ms, TE=8ms) exhibited abnormal high-intensity signals in the quadriceps femoris [vastus lateralis (VL) and vastus intermedius (VI)], adductor magnus (ADM), gluteus maximus (GM), biceps femoris long head (BFL), biceps femoris short head (BFS), semitendinosus (ST), soleus, lateral gastrocnemius (GCL), and medial gastrocnemius (GCM), predominantly on the right side. The peroneus longus (PL) exhibited abnormal T1 signals on the left side (A-C, transverse sections at the hip, thigh, and calf levels, respectively). The shaded areas in the illustrations indicate abnormal muscles of the right lower extremity in each section. A T2-weighted image ( $D, T R=3,600 \mathrm{~ms}$, TE=101ms) exhibited high-intensity signals with a similar distribution to that in the T1-weighted image (A), but normal short tau inversion recovery signals (E). Abbreviations: T1WI: T1-weighted image, T2WI: T2-weighted image, STIR: short tau inversion recovery, ADB: adductor brevis, ADL: adductor longus, EDL: extensor digitorum longus, GR: gracilis, IL: iliopsoas, PE: pectineus, RF: rectus femoris, SA: sartorius, SM: semimembranosus, TA: tibialis anterior, TP: tibialis posterior, VM: vastus medialis

\section{Discussion}

The case of PRLMNS reported herein developed 17 years after radiation therapy. Recurrence of germinoma and neoplastic infiltration at the nerve root or plexus were excluded based on the clinical course, neuroimagings, and cerebrospinal fluid findings (11). Multifocal motor neuropathy, which predominantly affects the distal upper limb, was excluded, because: (i) his symptoms were limited to the lower limbs for 13 years after the onset, (ii) there was no evidence of conduction block, and (iii) serum anti-ganglioside antibodies were absent, including anti-GM1 IgM antibody. The diagnosis of PRLMNS was supported by the typical clinical manifestation of delayed and progressive motor symptoms. Our patient received radiation greater than $65 \mathrm{~Gy}$ at the middle segment of the spinal cord, which exceeds the cutoff dose of 40 Gy suggested to cause PRLMNS (2). The observed needle EMG findings, in particular the myokymic discharges, are characteristic of PRLMNS $(11,12)$. The mild elevation of serum CK was interpreted to be secondary to denervation rather than a primary myopathic process. Mild hyperCKemia was also reported in a previous study of PRLMNS (7). Additionally, we found reduced SNAP amplitude of the sural nerve, which suggests sensory nerve damage distal to the dorsal root ganglion. This may have been due to subclinical toxic axonal neuropathy related to previous treatment with vincristine.

Even though PRLMNS was first reported in 1948 by Greenfield and Stark (3), the literature on PRLMNS remains 
very limited. Bowen et al. examined an autopsy case of PRLMNS and concluded that ischemic injury of the cauda equina nerve roots is caused by radiation-induced small vessel vasculopathy (2). However, the cauda equina pathology is often difficult to identify in vivo. In the present case, the conduction study of the tibial nerve showed mild delay of minimum F-wave latency and normal conduction velocity of the distal motor nerve. These results suggest focal proximal demyelination. However, by using the conventional methods, it is hard to further localize the lesion site. High-voltage electric stimulation at different vertebral levels can be used to detect proximal conduction delay at the cauda equina, but this procedure induces some degree of pain (13). The MATS coil is much better tolerated and it has been successfully applied to measure the CECT in chronic inflammatory demyelinating polyradiculoneuropathy and polyneuropathy, organomegaly, endocrinopathy, M-protein, and skin changes (POEMS) syndrome $(10,14)$. In the present study, primary damage in the cauda equina was suggested by a markedly prolonged CECT. In addition, it is likely that the cauda equina damage caused secondary axonal degeneration and remyelination of the motor nerves, because the CMAPs induced by proximal stimulation showed temporal dispersion and reduced amplitude (bottom 2 traces, Fig. 4) and active denervation potentials were revealed on needle EMG.

It is unknown why motor disturbances are predominant and sensory symptoms are often mild in PRLMNS. It may be explained by the differences in sensitivity and vulnerability between the sensory and motor nerves, presumably associated with factors such as ischemic tolerance and vascularization level. However, it should be noted that sensory involvement was previously suggested in PRLMNS (2). In addition, in the present case, delayed SSEP and normal peripheral conduction time suggested damages in the sensory system proximal to the dorsal root ganglion.

Skeletal muscle MRI was also found to be useful for the assessment of PRLMNS in the present case. The pattern of abnormal MRI signals (high intensity on T1- and T2weighted images and isointensity on STIR images) indicates chronic neurogenic changes with fat replacement in the lower extremity muscles $(15,16)$. Denervated muscles were characterized by a patchy distribution that deviated from the myotomal innervation patterns (17). The distribution was not compatible with combined peripheral nerve lesions but suggestive of patchy nerve root injuries within the cauda equina as reported previously $(2,7,8)$.

The present and previous results together indicate that PRLMNS is characterized by motor impairment and mild (or no) sensory symptoms. This clinical presentation differs from that of cauda equina syndrome with other causes, such as compression, trauma, and inflammation, which commonly manifest sensory and autonomic symptoms, including saddle anesthesia and bladder dysfunction. The CECT measure and skeletal muscle MRI may be useful for the early detection of the latent syndrome and is suitable for application in prospective longitudinal cohort studies.

The authors state that they have no Conflict of Interest (COI).

\section{Acknowledgement}

The authors are grateful to Dr. Toshimichi Maeda (Toranomon Hospital) for providing clinical information on the past treatment for germinoma received by the reported patient.

\section{References}

1. Reagan TJ, Thomas JE, Colby MY Jr. Chronic progressive radiation myelopathy. Its clinical aspects and differential diagnosis. JAMA 203: 106-110, 1968.

2. Bowen J, Gregory R, Squier M, Donaghy M. The post-irradiation lower motor neuron syndrome neuronopathy or radiculopathy? Brain 119: 1429-1439, 1996.

3. Greenfield MM, Stark FM. Post-irradiation neuropathy. Am J Roentgenol Radium Ther 60: 617-622, 1948.

4. De Carolis P, Montagna P, Cipulli M, Baldrati A, D’Alessandro R, Sacquegna $\mathrm{T}$. Isolated lower motoneuron involvement following radiotherapy. J Neurol Neurosurg Psychiatry 49: 718-719, 1986.

5. Gallego J, Delgado G, Tunon T, Villanueva JA. Delayed postirradiation lower motor neuron syndrome. Ann Neurol 19: 308-309, 1986.

6. Lamy C, Mas JL, Varet B, Ziegler M, de Recondo J. Postradiation lower motor neuron syndrome presenting as monomelic amyotrophy. J Neurol Neurosurg Psychiatry 54: 648-649, 1991.

7. van der Sluis RW, Wolfe GI, Nations SP, et al. Post-radiation lower motor neuron syndrome. J Clin Neuromuscul Dis 2: 10-17, 2000.

8. Ducray F, Guillevin R, Psimaras D, et al. Postradiation lumbosacral radiculopathy with spinal root cavernomas mimicking carcinomatous meningitis. Neuro Oncol 10: 1035-1039, 2008.

9. Matsumoto H, Octaviana F, Terao Y, et al. Magnetic stimulation of the cauda equina in the spinal canal with a flat, large round coil. $\mathrm{J}$ Neurol Sci 284: 46-51, 2009.

10. Matsumoto H, Hanajima R, Terao $\mathrm{Y}$, et al. Prominent cauda equina involvement in patients with chronic inflammatory demyelinating polyradiculoneuropathy. J Neurol Sci 290: 112-114, 2010.

11. Thomas JE, Cascino TL, Earle JD. Differential diagnosis between radiation and tumor plexopathy of the pelvis. Neurology 35: 1-7, 1985.

12. Albers JW, Allen AA 2nd, Bastron JA, Daube JR. Limb myokymia. Muscle Nerve 4: 494-504, 1981.

13. Ugawa Y, Genba-Shimizu K, Kanazawa I. Electrical stimulation of the human descending motor tracts at several levels. Can J Neurol Sci 22: 36-42, 1995.

14. Matsumoto H, Hanajima R, Terao Y, Hashida H, Ugawa Y. Neurophysiological analysis of the cauda equina in POEMS syndrome. Neurol Sci 34: 121-122, 2013.

15. Kamath S, Venkatanarasimha N, Walsh MA, Hughes PM. MRI appearance of muscle denervation. Skeletal Radiol 37: 397-404, 2008.

16. West GA, Haynor DR, Goodkin R, et al. Magnetic resonance imaging signal changes in denervated muscles after peripheral nerve injury. Neurosurgery 35: 1077-1085, 1994.

17. Kim SJ, Hong SH, Jun WS, et al. MR imaging mapping of skeletal muscle denervation in entrapment and compressive neuropathies. Radiographics 31: 319-332, 2011.

(C) 2015 The Japanese Society of Internal Medicine http://www.naika.or.jp/imonline/index.html 